\title{
Models Having Tunable Parameters in Scale-free Networks
}

\author{
Fei Ma, Jing Su, Bing Yao \\ College of Mathematics and Statistics, Northwest Normal \\ University, Lanzhou, 730070, China \\ E-mail: yybb918@163.com
}

\author{
Ming Yao \\ Department of Information Process and Control \\ Engineering, Lanzhou Petrochemical College of \\ Vocational Technology Lanzhou, 730060, China \\ E-mail: yybm918@163.com
}

\begin{abstract}
Based on typical scale-free network models (BA-model), we set up the partial differential equation satisfied more general dynamic networks. Starting from a more general situation, we establish two network models and show their topological properties that obey power laws.
\end{abstract}

Keywords-scale-free models; dynamical evolution equation; small-world effect; social network

\section{INTRODUCTION}

Many complex systems can be depicted as network models. In the past twenty years we have witnessed an upsurge in the research of a wide range of complex systems which can be described in terms of networks--vertices connected together by edges. Small-world effect and Scale-free property are common in most real-life networks (Ref. [6, 7, 9, 17, 20, 21, 22], [26-30]), such as the Internet, protein-protein interactions Ref.[10, 16]), transportation systems or social and economic networks and so on. Complex networks with these two characteristics are called small-world scale-free networks, where scale-free networks obey $P(k) \approx k^{-\gamma}$ for the scale-free parameter $\gamma$ falling into $2<\gamma<3$ (Ref.[19]). There are three steps on studying complex systems and networks. The first step is in the 1950s, based on researching the classic the graph theory, Erdos and Renyi (Ref.[14]), as the pioneers of this investigation, came up with the stochastic network model (ER-model) to describe complex network. The second step can be traced to Watts and Strogatz (Ref.[2, 4]) put forward the small-world model (WS-model) in 1998. The third step is due to Barabasi and Albert (Ref.[3]). By researching the topological structure of WWW they presented firstly the scale-free network model (BA-model) in 1999. It is so clear for us that every step has itself focus. The researching way has been pushed from "regualtion and stochastical" to "the complex". In a words, three networks models mentioned above can not describe better numbers of real-life networks having small-world effect and scale-free property.

It is an urge research project how to design some better models. Taking three representative models into account, researchers generate abundant various models, each one is structured with individual angle, which have scale-free property no small-world effect like stochastic models: BA scale-free two-way evolution network models (Ref.[26]), an evolving model for scale-free collaboration networks (Ref.[28]\}), a dynamic scale-free network model (Ref.[22]), etc. Apparently, these new vertices of each model are added into networks in isolation in this dynamical process. The result leads to it with lower cluster coefficient. For obtaining higher one, some studier build some deterministic models like Apollonian models, Farey graph models, Sierpinski models (Ref.[8]), and so on. These models have small-world effect but no randomly generating. The dynamical evolution of most real-life networks is random and free. So them are not ideal ones. The writer (Ref.[27]) says a stochastic model named group growing model based small-world scale-free network which has these two characteristics.

In this paper. Firstly, we establish the dynamical partial differential equation relative the dynamical evolution process of complex systems and networks. Secondly, we design one class tuned parameters small-world scale-free network Models by improving the simple entering way of adding some new isolate vertices into networks.

\section{DYNAMICAL PARTIAL DIFFERENTIAL EQUATION}

Making some descriptions and states for the dynamical evolution process, which contains some new vertices entered continuously, some new edges generated continuously, many old vertices removed continuously, many old edges removed continuously and much interference from the outside at each time step. There exists lots of similarity about these under all almost evolutions happening under this given suppose preferential attachment mechanism. As time goes on, the scope of dynamical complex network become more and more huger by these discussions. In the other word, the number vertices and edges increase and its spacial structure will become more complex. Nothing will always grow unceasingly. Undergoing after an extended period, network itself will tend to a stable or decay.

We may suppose the dynamic evolution of a connected original network $N(0)$ having $m_{0}$ vertices is continual and then can set up a corresponding partial differential equation to explain this dynamical system, according to the independence of events. There is a corresponding partial differential equation for a degree $k_{i}(t)$ vertex in the network $N(t)$ at time step t shown below. Following

$$
\frac{\partial k_{i}(t)}{\partial t}=f^{*}(t)+g^{*}(t)+h^{*}(t)+z^{*}(t)+\varphi(t)
$$

where (i) The entering vertices function $f^{*}(t)=f\left(a p_{1}(t) m, t\right.$, $\left.k_{i}(t), \Sigma \Pi_{1 j}\left(k_{i}\right)\right)$ indicates there are a new vertices entered in the network $N(t-1)$ at time step $t$. The new vertex $j_{a}$ will devote $p_{1}(t) m\left(0<p_{1}(t)<1\right)$ edges into $N(t-1)$ by connecting with some old vertices. The probability which the degree $k_{i}(t)$ vertex obtains new links obeys the preferential attachment probability $\Pi_{1 j}\left(k_{i}\right) . \Sigma \Pi_{1 j}\left(k_{i}\right)$ stands for all kinds of probability 
co-existing (the most important is preferential attachment probability). (ii) The removing vertices functio $g^{*}(t)=g\left(p_{2}(t) b\right.$, $\left.t, k_{i}(t), \Sigma \Pi_{2 j}\left(k_{i}\right)\right)$, after network goes through the quickly increasing process, shows there are $p_{2}(t) b\left(0<p_{2}(t)<1\right)$ old vertices removed from the network $N(t-1)$ at time step $\mathrm{t}$. An old vertex $j_{b}$ will be removed with the probability $\Pi_{2 j}\left(k_{i}\right)$ (the opposite-preferential attachment probability). (iii) The adding edges function $h^{*}(t)=h\left(p_{3}(t) r, \quad t, \quad k_{i}(t), \quad \Sigma \Pi_{3 j}\left(k_{i}\right)\right)$ suggests there will be $p_{3}(t) r\left(0<p_{3}(t)<1\right)$ "new"edges added among a few pairs of vertices which are not linked in the network $N(t-1)$ at time step $t$. The two vertices linked by one new edge $j_{r}$ will be selected with the preferential attachment probability $\Pi_{3 j}\left(k_{i}\right)$. (iv) The canceling edges function $z^{*}(t)=z\left(p_{4}(t) s, t, k_{i}(t), \Sigma \Pi_{4 j}\left(k_{i}\right)\right)$ implies $p_{4}(t) s\left(0<p_{4}(t)<1\right)$ old edges will be canceled at time step $t$. In the process, the two vertices linked by a canceled old edge $j_{s}$ will be selected with the opposite-preferential attachment probability $\Pi_{4 j}\left(k_{i}\right)$. (v) The external disturbance function $\varphi(t)$ hints the process of networks evolution will endure unavoidable influence from the outside world.

\section{A. First Model}

The constructing process of $N_{1}(t)$ is: The original network $N_{1}(0)$ is a connected simple graph having $n_{0}$ vertices and $m_{0}$ edges. From time step $t \geq 1$, two operations below will be carry out. (i) Growth. At time step $t$, there is a group new vertices, including $2 a$, entered into the network $N_{1}(t-1)$. (ii) The Preferential Attachment Operation. This group vertices are made up two classes different vertices. The number of the others is $2 \alpha a$, another is $2(1-\alpha) a$. Each vertex of the first part links randomly $m\left(\leq m_{0}\right)$ old vertices in $N_{1}(t-1)$ by using the preferential attachment probability $k_{i} / \Sigma k_{j}$. The connecting way is named star-graph-growth-way. Any two vertices of the second part will generate a new edge by link each other. The operation leads to $(1-\alpha) a$ new edges. The two vertices of a new edge will simultaneously link randomly $m-1\left(\leq m_{0}\right)$ old vertices in $N_{1}(t-1)$ by using the preferential attachment probability $k_{i} / \Sigma k_{j}$. This connecting way is named triangle-growth-way. This growth process may circularly go on until an expected model is captured.

Apparently, any edge of the second part links randomly $m-1\left(\leq m_{0}\right)$ old vertices in $N_{1}(t-1)$ will bring $m-1$ triangles $\left(K_{3}\right)$, it shows a man having a old friend make friends with $\mathrm{m}-1$ unknown persons and then introduce his old friend to those new friends just known soon by the way. It is clear for us that the network will add $(1-\alpha) a m$ triangles at every time step which emerges higher clustering effect in local social re-communication network. Abstractly, a mass of higher dense triangles will appear in topological structure of the network. Obviously, the whole network clustering coefficient has a lager enhancing than those presented scale-free models by tuning the number of higher dense triangles. The small-world property come true. Abundant deterministic scale-free network models, published in the last ten years, all adopt this growth way (triangle-growth-way) to obtain small-world property. The advantage our model is stochastic can be considered as a better model to simulate some character properties of complex system in the future.
After couple time steps, the network model $N_{1}(t)$ has $n_{v}(t)=n_{0}+2 a t$ vertices and $n_{e}(t)=m_{0}+[2 a m-(1-\alpha) a] t$ edges. It is easy for us that it will be a BA-model if the parameter $\alpha=1$ is true. Assuming that $k_{i}$ is a continuous real variable. According to its dynamical evolution mechanism

$$
f^{*}(t)=\frac{2 \alpha a m k_{i}+\left[(1-\alpha) a(m-1)^{2}\right] k_{i}}{2 m_{0}+2[2 a m-(1-\alpha) a] t}
$$

Others functions $g^{*}(t)=h^{*}(t)=z^{*}(t)=\varphi(t)=0$. Consequently, $k_{i}$ satisfies the dynamical equation

$$
\frac{\partial k_{i}(t)}{\partial t}=Q_{1} \frac{k_{i}}{t}, Q_{1}=\frac{2 \alpha a m+(1-\alpha) a(m-1)^{2}}{2[2 a m-(1-\alpha) a]}
$$

The solution of the equation (2), with the initial condition that each vertex $i$ at its introduction has $k_{i}\left(t_{i}\right)=m$, is $k_{i}(t)=m\left(t / t_{i}\right)^{Q_{1}}$. The probability that one vertex has a degree $k_{i}(t)$ smaller than $k\left(P\left(k_{i}(t)<k\right)\right)$ can be written as

$$
P\left(k_{i}(t)<k\right)=P\left(t_{i}>t \frac{m^{1 / Q_{1}}}{k^{1 / Q_{1}}}\right)=1-P\left(t_{i} \leq t \frac{m^{1 / Q_{1}}}{k^{1 / Q_{1}}}\right)
$$

Supposing which we add these new vertices at equal time interval into the network, namely $P\left(t_{i}\right)=1 /\left(m_{0}+t_{i}\right)$. Set $M_{1}(k)=m^{1 / Q_{1}} / k^{1 / Q_{1}}$, then

$$
P\left(t_{i} \leq t \frac{m^{1 / Q_{1}}}{k^{1 / Q_{1}}}\right)=\int_{0}^{M_{1}(k)} P\left(t_{i}\right) d t=\frac{M_{1}(k)}{m_{0}+t_{i}}
$$

The degree distribution $P(k)$ can be computed as

$$
P(k)=\frac{\partial P\left(k_{i}(t)<k\right)}{\partial k}=\frac{t \cdot m^{1 / Q_{1}}}{\left(m_{0}+t\right) Q_{1} \cdot k^{1 / Q_{1}+1}},
$$

$P(k) \approx \frac{m^{1 / Q_{1}}}{Q_{1}} k^{-\left(1 / Q_{1}+1\right)}(t \rightarrow \infty)$

It obeys the power law with parameter

$$
\gamma=1+1 / Q_{1}=1+\frac{2[2 m-(1-\alpha)]}{2 \alpha m+(1-\alpha)(m-1)^{2}}
$$

If $\alpha=1$, then $\gamma=3$. If $\alpha=0$, then $\gamma=(4 m-2) /(m-1)^{2}+1$, and as $m \rightarrow \infty,(4 m-2) /(m-1)^{2} \rightarrow 0$ is true. If $\alpha=1 / 2, m=3$, then $\gamma=(8 m-2) /\left[2 \mathrm{~m}+(m-)^{2}\right]+1=3.2$. The value range of the power law parameter $\gamma$ is not relative with the parameter $a$. That $1<\gamma<7$ is true. We calculate

$$
\sum\left|V\left(k_{i}, t\right)\right| \frac{\partial k_{i}(t)}{\partial t}=\sum \frac{2 \alpha a m+(1-\alpha) a(m-1)^{2}}{2 m_{0}+2[2 a m-(1-\alpha) a] t} k_{i}(3)
$$

The symbol $\left|V\left(k_{i}, t\right)\right|$ stands the number of vertices having degree $k_{i}$ at time step $t$. It is evident for us that this equation (3) attend to a constant value $2 \alpha a m+(1-\alpha) a(m-1)^{2}$. For some researchers working the network security, they want to obtain the number of hub-vertices(Ref.[18, 24]) after getting the 
degree distribution $P(k)$, because they can protect it from interference by control some hub-vertices of the whole network. Next, we use the cumulative formula introduced in [5] to get that the model $N_{1}(t)$ holds

$$
P_{\text {cum }}^{1}(k)=\frac{1}{n_{v}(t)} \sum_{k^{\prime} \geq k}\left|V\left(k^{\prime}, t\right)\right| \approx-\int_{k}^{n_{v}(t)-1} P\left(k^{\prime}\right) d k \approx l_{1}^{1 / Q_{1}} k^{-\left(1 / Q_{1}+1\right)}
$$

which implies that $N_{1}(t)$ is scale-free.

\section{B. Second Model}

The second model $N_{2}(t)$ can be build up in the way: The initial network $N_{2}(0)$ is a connected imple graph having $n_{0}$ vertices and $m_{0}$ edges. From time step $t \geq 1$, these two operations below will be carry out. (i) Growth. At time step t, there is a group new vertices, including $2 \mathrm{a}$, entered into the network $N_{2}(t-1)$. (ii) The Preferential Attachment Operation. This group vertices are made up two classes different vertices. The number of the first part is $2 \alpha a$, another is $2(1-\alpha) a$. Each vertex of the first part links randomly $m\left(\leq m_{0}\right)$ old vertices in $N_{2}(t-1)$ by using the preferential attachment probability $k_{i}$ $/ \Sigma k_{j}$. The second part contains two classes different vertices, A-class and B-class. Any two vertices of the second part will generate a new edge by link each other. The operation leads to $(1-\alpha) a$ new edges. The two vertices of a new edge of the A-class, including $2 \beta(1-\alpha) a$ vertices, will simultaneously link randomly $m-1\left(\leq m_{0}\right)$ old vertices in $N_{2}(t-1)$ by using the preferential attachment probability $k_{i} / \Sigma k_{j}$. Every vertex of a new edge of the B-class, including $2(1-\beta)(1-\alpha) a$ vertices, will link randomly $m-1\left(\leq m_{0}\right)$ diverse old vertices in $N_{2}(t-1)$ by using the preferential attachment probability $k_{i} / \Sigma k_{j}$. This growth process may circularly go on until an expected model is captured. Obviously, any edge of the A-class links randomly $m-1\left(\leq m_{0}\right)$ old vertices in $N_{2}(t-1)$ will bring $m-1$ triangles $\left(K_{3}\right)$ which shows a man having a old friend make friends with $m-1$ unknown persons and then introduce his old friend to those new friends just known soon by the way. Clearly, the network model $N_{2}(t)$ has $n_{v}(t)=n_{0}+2 a t$ vertices and $n_{e}(t)=m_{0}+[2 a m-(1-\alpha) a] t$ edges at time step $t$.

Assuming that $k_{i}$ is a continuous real variable. According to its dynamical evolution mechanism

$$
f^{*}(t)=\frac{[2 \alpha a+(1-\beta)(1-\alpha) a] m+\left[\beta(1-\alpha) a(m-1)^{2}\right]}{2 m_{0}+2[2 a m-(1-\alpha) a] t} k_{i}(4)
$$

Others functions $g^{*}(t)=h^{*}(t)=z^{*}(t)=\varphi(t)=0$. Consequently, $k_{i}$ satisfies the dynamical equation

$$
\begin{aligned}
& \frac{\partial k_{i}(t)}{\partial t}=Q_{2} \frac{k_{i}}{t} \\
Q_{2}= & \frac{[2 \alpha a+(1-\beta)(1-\alpha) a] m+\left[\beta(1-\alpha) a(m-1)^{2}\right]}{2[2 a m-(1-\alpha) a]}
\end{aligned}
$$

Since each vertex $i$ at its introduction has $k_{i}\left(t_{i}\right)=m$, thus, we solve the equation (4), and get the solution $k_{i}(t)=m\left(t / t_{i}\right)^{1 / Q_{2}}$. The probability $P\left(k_{i}(t)<k\right)$ that one vertex has a degree $k_{i}(t)$ smaller than $k$ is

$$
P\left(k_{i}(t)<k\right)=P\left(t_{i}>t \frac{m^{1 / Q_{2}}}{k^{1 / Q_{2}}}\right)=1-P\left(t_{i} \leq t \frac{m^{1 / Q_{2}}}{k^{1 / Q_{2}}}\right)
$$

Supposing that we add these new vertices at equal time interval into the network, namely $P\left(t_{i}\right)=1 /\left(m_{0}+t_{i}\right)$. Set $M_{2}(k)=m^{1 / Q_{2}} / k^{1 / Q_{2}}$, we have

$$
P\left(t_{i} \leq t \frac{m^{1 / Q_{2}}}{k^{1 / Q_{2}}}\right)=\int_{0}^{M_{2}(k)} P\left(t_{i}\right) d t=\frac{M_{2}(k)}{m_{0}+t_{i}}
$$

Thereby, the degree distribution $P(k)$ is equal to

$$
P(k)=\frac{\partial P\left(k_{i}(t)<k\right)}{\partial k}=\frac{t \cdot m^{1 / Q_{2}}}{Q_{2}\left(m_{0}+t\right)} k^{-\left(1 / Q_{2}+1\right)} \approx \frac{m^{1 / Q_{2}}}{Q_{2}} k^{-\left(1 / Q_{2}+1\right)}(t \rightarrow \infty)
$$

The network model $N_{2}(t)$ obeys the power law with parameter

$$
\gamma=1+1 / Q_{2}=1+\frac{2[2 m-(1-\alpha)]}{[2 \alpha+(1-\beta)(1-\alpha)] m+\beta(1-\alpha)(m-1)^{2}}
$$

If $\alpha=\beta=1$, then $\gamma=3$. If $\alpha=0, \beta \neq 0$, then $\gamma=(4 m-2) /\left[(1-\beta) m+\beta(m-1)^{2}\right]+1$, and as $m \rightarrow \infty$, $(4 m-2) /\left[(1-\beta) m+\beta(m-1)^{2}\right] \rightarrow 0$ is true. If $\alpha=\beta=1 / 2, m=4$, then $\gamma=(8 \mathrm{~m}-2) /\left[5 \mathrm{~m} / 2+(m-1)^{2} / 2\right]+1=89 / 29>3$. If $\alpha=1 / 2, \beta=1 / 4$, $m=4$, then $\gamma=(8 \mathrm{~m}-2) /\left[11 \mathrm{~m} / 4+(m-1)^{2} / 4\right]+1=173 / 53>3$. If $\alpha=1 / 2, \quad \beta \quad=3 / 4, \quad m=4, \quad$ then $\gamma=(8 \mathrm{~m}-2) /\left[9 \mathrm{~m} / 4+3(m-1)^{2} / 4\right]+1=183 / 63<3$. The value range of the power law parameter $\gamma$ is not relative with the parameter $a$. However, $1<\gamma$ is true. We, for $N_{2}(t)$, come to compute

$$
\sum V\left(k_{i}, t\right) \mid \frac{\partial k_{i}(t)}{\partial t}=\sum \frac{[2 a a+(1-\beta)(1-\alpha) a] m+\beta(1-\alpha)(m-1)^{2}}{2 m_{0}+2[2 a m-(1-\alpha) a] t} k_{i}(5)
$$

The symbol $\left|V\left(k_{i}, t\right)\right|$ stands the number of vertices having degree $k_{i}$ at time step $t$. Hence, (5) approaches to $[2 \alpha a+(1-\beta)(1-\alpha) a] m+\beta(1-\alpha) a(m-1)^{2}$ as $t$ runs to infinity. For some researchers working the network security, they want to obtain the number of hub-vertices after getting the degree distribution $P(k)$, because they can protect it from interference by control some hub-vertices of the whole network (Ref. [18, 24]). By the cumulative formula introduced in [5], we say the model $N_{2}(t)$ is scale-free, since

$$
P_{\text {cum }}^{2}(k)=\frac{1}{n_{v}(t)} \sum_{k^{\prime} \geq k}\left|V\left(k^{\prime}, t\right)\right| \approx-\int_{k}^{n_{v}(t)-1} P\left(k^{\prime}\right) d k \approx l_{2}{ }^{1 / Q_{2}} k^{-\left(1 / Q_{2}+1\right)}
$$

\section{SUMMARY}

According to a mass of research results about complex systems and networks at present, we firstly set up the general partial differential equation with five characteristic functions and achieve its solutions by abstract numerical analysis under a special initial condition. In this dynamical evolution process, we apply the degree-preferential-attachment mechanism (Ref. [23]) to make the model be scale-free and connect one part new adding vertices with edges to form masses of higher dense triangles for letting it be small-world. 
At the same time, the procedure of creating our models is so easy to do and achieve that we can build network models with the realistic topological structure, then explain and estimate its characters by simulating complex networks growing process. Our model may provide a useful tool to investigate the influence of the clustering coefficient or average path length in different dynamics processes taking place on networks. In addition, The following some meaningful questions as the research in the future remain open (Ref. [15, 25]).

\section{ACKNOWLEDGEMENT}

We are grateful to the anonymous referees for their valuable and help comments which lead to the improvement of the paper. The author, Bing Yao, was supported by the National Natural Science Foundation of China under grants Nos 61163054, 61363060, 61662066. The author, Ming Yao, was supported by the Special Funds of Finance Department of Gansu Province of China under Grant No. 2014-63.

\section{REFERENCES}

[1] J. A. Bondy and U. S. R. Murty. Graph Theory with Application. The MaCmillan Press Itd, London and Basingstoke, New York, 1976.

[2] D. J. Watts and S. H. Strogatz. Collective dynamics of small-world networks, Nature 393(1998)

[3] A. -L. Barabsi, R. Albert, H. Jeong. Meaneld theory for scale-free random networks. Physica A 272 (1999) 173-187.

[4] D. J. Watts. Small Worlds: The Dynamics of Networks between Order and Randomness. Princeton University Press, Princeton, NJ, 1999.

[5] M. E. J. Newman. The structure and function of complex networks. J. SIAM Review 45 (2003) 167-256.

[6] Z. Z. Zhang, S. Zhou, L. Fang, J. Guan. Y. Zhang. Maximal planar scale-free Sierpinski networks with small-world effect and power law strength-degree correlation. Europhys, Lett. 79 (2007) 38007.

[7] S. C. Chang, L. C. Chen, W. S. Yang. Spanning trees on the Sierpinskigasket. J. Stat. Phys. 126 (2007) 649-667.

[8] Z. Z. Zhang, S. Zhou. Collerations in random Apollonian networks. J. Physica A 380 (2007) 621-628.

[9] F. Comellas. Z. Zhang, L. Chen. Self-similar non-clustered planar graphs as models for complex networks. J. Phys. A: Math. Theor. 42 (2009) 045103 .

[10] A. Miralles, F. Comellas, L. C. Chen, Z. Z. Zhang. Planar unclustered scale-free graphs as models for technological and biological networks. J. Phys. 389 (2010) 1995-19964.

[11] E. Teu, S. Wagner. Resistance scaling and the number of spanning trees in self-similar lattices. J. Stat. Phys. 142 (2011) 879-897.

[12] F. Comellas, A. Miralles, H. X. Liu, Z. Z. Zhang. The number of spannings trees of an innite family of outerplannar, small-world and self-similar graphs. J. Physica A 392 (2013) 2803-2806.

[13] G. Yan, G. Tsekenis, B. Barzel, J. JSlotine, Y. Y. Liu, A. L. Barabasi. Spectrum of controlling and observing complex networks. J. Nature Physics. 3422 (2015) 779-786.

[14] Erdos P, R enyi A. On random graphs. Publ. Math., 1959, 6: 290-297

[15] B. Yao, M. Yao, X, E, Chen, X. Liu, W. J. Zhang, Research on Edge-Growing models related with scale-free small-world networks, J, Applied Mechanics and Materials, Volumes 513-517, 2014

[16] C. M. Song, T. Koren, P. Wang, A. L. Barab asi, Modelling the scaling properties of human mobility, J. Nature Physics. 1760 (2010) 1-6

[17] H. G. Liu, S. H. Cai, Y. Q. Zhang. The research on the scale-free networks model, J. College Physics, 2008 (4)
[18] G. Yan, G. Tsekenis, B. Barzel, J. JSlotine, Y. Y. Liu, A. L. Barab asi, Spectrum of controlling and observing complex networks, J. Nature Physics. 3422 (2015) 779-786

[19] Charo. I. Del. Genio, Thilo. Gross, Kevin. E. Bassler, All scale-free network are sparse, J. Physical review letters. 178701 (2011) 1-4

[20] Q. H. Chen, D. H. Shi. the modeling of scale-free networks, J. Physica A, 2004, 335:240-248

[21] H. Z. Liang, H. X. Yao, X. B. Zhang. Characters Analysis for a Class of Scale-Free Networks, J. Complex Systems and Complexity Science, 2005 (3)

[22] X. L. Jia, S. H. Cai, F. R. Zhang. A Dynamic Scale-free Network Model, J. Journal of Sichuan Normal University (Natural Science), 2009 (6)

[23] P. L. Krapivsky, S. Redner, F. Leyvraz, Connectivity of growing random network, J. Phys. Rev. Lett. 85,5901(2000)

[24] L. Wang, G. Z. Dai. On Degree Distribution of Complex Network, J. Journal of Northwestern Polytechnical University, 2006 (4)

[25] B. Yao, X. Liu, W. J. Zhang, X. E. Chen, X. M. Zhang, M.Yao, Z. X. Zhang. 2013 IEEE International Conference on High Perfermance Computing and Communication and 2013 IEEE International Conference on Embedded and Ubiquiyous Computing, 2354-2361. B. Yao, M. Yao, X. E. Chen, X. Liu, W. J. Zhang. Applied Meachanics and Materials,Vol. 513-517,2013, 2444-2448. B. Yao, C. Yang, M. Yao, H. Y. Wang, X. E. Chen, X. M. Zhang, M. G. Li. Applied Mechanics and Materials, Vol.380-384(2013) 2034-2037. DOI: 10.4028/www.scientic.net/AMM.380- 384.2034. B Yao, X Liu, X-m Wang, X-y Zhao, M Yao. Proceedings of the 2015 International Symposium on Computers \& Informatics, ISBN (on-line): 978-94-62520-56-1, volume 13.pp1567-1574.

[26] F. Q. Gu, S. H. Fan. The two-way evolution of a BA scale-free network, J. Journal of Jinan University (Natural Science), 2013 (5)

[27] A. Wu, X. S. Liu, D. Liu, C. S. Zuo. Group Growing Based Small-World Scale-Free Network Model, J. Journal of Computer Science, 2005 (7)

[28] Z. Z. Zhang, B. Wu, F. Comellas. J. Discrete3 Applied Mathematics. 169 (2014) 206-213. Z. Z. Zhang, L. L. Rong, T. Zhou. J. System Engineering-Theory Practice, 2005 (25). Z. Z. Zhang, B. Wu, Y. Lin. J. Physica A, 391(2012) 3342-3349. Z. Z. Zhang, S. G. Zhou, Z. Su, T. Zhou, J. H. Guan. J. Nature 2008.

[29] X-m Wang, X-y Zhao, B Yao, M Yao, X-e Chen. Applied Mechanics and Materials(AMM) ISSN print 1660-9336 ISSN cd 1660-9336 ISSN web 1662-7482. X-m Wang, B Yao, F Ma, J Su, X-e Chen, M Yao. 2015 12th International Conference on Fuzzy Systems and Knowledge Discovery (FSKD'15). 978-1-4673-7681-5 C2015 IEEE 476-481. X-m Wang, B Yao, F Ma, X-y Zhao, J Su, X-e Chen, M Yao. 2015 8th International Conference on BioMedical Engineering and Informatics (BMEI 2015)783-788. X-m Wang, X-y Zhao, B Yao, X Liu, X-e Chen. 2015 2nd International Conference on Technological Advances in Electrical, Electronics and Computer Engineering (ICCNEE 2015) 1157-1162. X-m Wang, B Yao, M Yao. 170-173. IEEE Catalog Number: CFP16D86-ART, ISBN: 978-1-4673-9194-8.IEEE Catalog Number: CFP16D86-CDR, ISBN: 978-1-4673-9193-1.

[30] X Liu, B Yao, X-m Wang, X-y Zhao, M-j Zhang. On Evolution Properties of E-bound Growing Network Models. Proceedings of the 2015 International Symposium on Computers \& Informatics, ISBN (on-line): 978-94-62520-56-1, part of the series ACSR, ISSN 2352-538x, volume 13. pp1559-1566. 